\title{
Sistema de información gerencial y su influencia en la calidad del servicio: empresa TEMCORPSA S.A.
}

\section{Management information system and its influence on the quality of service: business TEMCORPSA S.A.}

\author{
Jorge Véliz Tamayo ${ }^{1}$ \\ jovelizt@gmail.com \\ https://orcid.org/0000-0001-7150-8432 \\ Lisset Cotto Aguilar ${ }^{2}$ \\ sulema_cotto@hotmail.es \\ https://orcid.org/0000-0001-5983-3419
}

Recibido: 28/9/2021, Aceptado: 28/12/2021

\begin{abstract}
RESUMEN
La investigación tiene como objetivo mejorar la calidad del servicio entregado a los clientes de la empresa TEMCORPSA S. Para identificar las debilidades en el servicio y cómo un sistema de información gerencial podría aportar a la solución del problema, se trabajó con la población conformada por el área de talento humano y los clientes de la organización, con los cuales se aplicó una metodología con alcance descriptivo de enfoque cualitativo y cuantitativo, la muestra utilizada fue de 10 trabajadores de la organización y 40 clientes. Como resultado se obtuvo que existen escasos esfuerzos por transmitir seguridad al cliente respecto al servicio contratado, baja capacidad de respuesta a sus requerimientos, promociones y beneficios que no generan suficiente interés en el público, retrasos en los procesos de atención al cliente, entre otras en donde también figura el no disponer de un registro de clientes efectivo que ayude a pronosticar la demanda y anticiparse a sus necesidades. Por tanto, el desarrollo de procesos que vayan en función de un incentivo a los trabajadores de la organización, a la vez que el personal técnico realice revisiones periódicas a los equipos tecnológicos, ocasionará una mejor atención y seguridad al servicio brindado al cliente.
\end{abstract}

Palabras clave: Servicio, cliente, calidad, tecnología, seguridad

${ }^{1}$ Magister en informática de gestión y nuevas tecnologías, Universidad Técnica Federico Santa María, Chile

${ }^{2}$ Magister en Sistemas de Información Gerencial, Universidad Tecnológica Empresarial de Guayaquil, Ecuador 


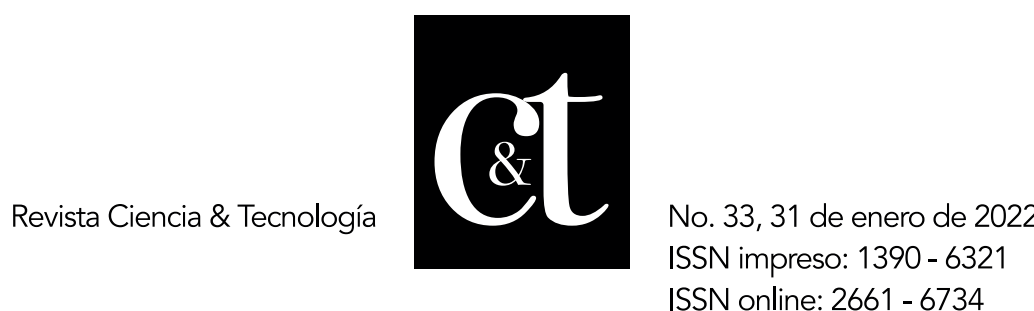

\begin{abstract}
The objective of the research is to improve the quality of the service delivered to the clients of the company TEMCORPSA S. To identify weaknesses in the service and how a management information system could contribute to solving the problem, we worked with the population made up of the Human talent area and the organization's clients, with which a methodology with a descriptive scope of qualitative and quantitative approach was applied, the sample used was 10 workers of the organization and 40 clients. As a result, it was obtained that there are few efforts to transmit security to the client regarding the contracted service, low capacity to respond to their requirements, promotions and benefits that do not generate enough interest in the public, delays in the customer service processes, among others in where there is also the lack of an effective customer registry to help forecast demand and anticipate their needs. Therefore, the development of processes that are based on an incentive to the workers of the organization, at the same time that the technical staff carry out periodic reviews of the technological equipment, will cause a better attention and security to the service provided to the client.
\end{abstract}

Keywords: Service, customer, quality, technology, security.

\section{Introducción}

El brindar un servicio de calidad al cliente debe ubicarse como el enfoque principal de las empresas. Sánchez (2018) expresó que este enfoque empieza a volverse más notable cuando existe un mayor interés en fidelizar al cliente, en cumplir sus expectativas y retenerlo. El interés en la fidelización surge debido a que el mercado se ve colapsado de productos, provocando que el cliente tenga más opciones de compra, la competencia se intensifique y reciba constantemente mensajes de otros oferentes. Vélez (2018) expresó que la fidelización del cliente parte de cómo una oferta lo satisface, diseñándola de tal forma que cumpla sus expectativas.

Las expectativas se construyen basadas en las referencias del cliente sobre un producto, mismas que deben ser cubiertas cuando lo adquiere y experimenta, lo cual vuelve relevante evaluar cuáles son estas expectativas para así lograr cubrirlas. De esta manera, en la medida que la experiencia iguale o supere las expectativas, se alcanzarán mayores niveles de satisfacción percibiéndose de mayor calidad y ello contribuirá a la fidelización del cliente. Hoces (2018) indicó que la fidelización consiste en el vínculo que una empresa o marca construye con sus clientes, los cuales preferirán su oferta en relación a la proporcionada por los competidores. Este vínculo se crea no solo cumpliendo las expectativas de los clientes, sino también mediante estrategias coordinadas, mismas que les proporcionen un valor agregado, respondan sus dudas o sugerencias como parte del servicio post venta, y creen así un vínculo emocional con éstos a largo plazo que se traducirá en un aumento de los ingresos percibidos para la entidad. 


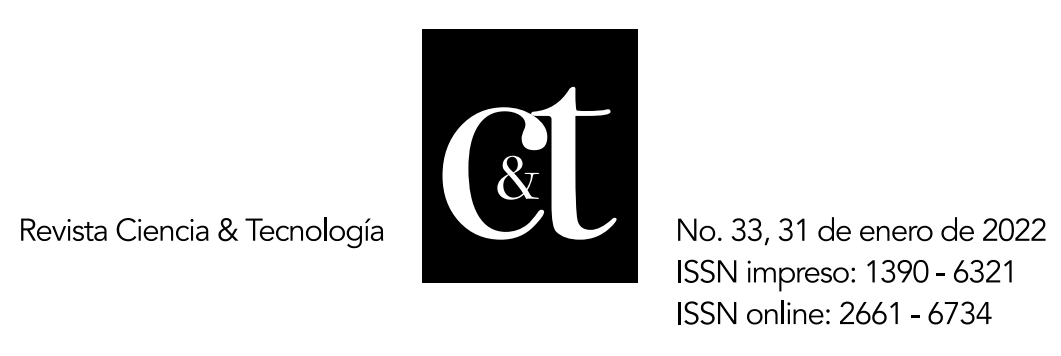

Este incremento obedece a que la empresa retendrá clientes, los cuales son menos sensibles a ofertas de la competencia y poseen más probabilidades de realizar compras futuras, incluso incrementarlas si la entidad continúa manteniendo su óptimo desempeño.

Alcaide (2015) expresó que, a pesar de la importancia de satisfacer a sus clientes, entregando un servicio de calidad y así fidelizarlos, son pocas las entidades que les proporcionan una oferta acorde a sus expectativas, misma que ha experimentado una reducción en sus ventas ocasionadas por la entrega de un servicio que no cumple las expectativas del público, percibido así de baja calidad, perdiendo clientes. Silva (2018) determina que mediante estos sistemas se recopilan y procesan datos a fin de producir información que posteriormente pueda ser utilizada en la planificación y control de las operaciones, inclusive para identificar las expectativas del cliente, logrando en su conjunto que una oferta sea percibida de mayor valor.

La calidad es subjetiva y depende de las expectativas y experiencias de cada individuo. Cortés (2017) indicó que la calidad en las organizaciones se asociaba directamente al producto y para garantizarla se realizaba una evaluación cuando el producto se encontraba elaborado. Mateos (2019) expresó que resulta esencial en las empresas que quieren alcanzar una posición reconocida en el mercado y mantenerse en el tiempo.

En relación a este tema, Arenal (2016) indicó que todo cliente tiene una expectativa al momento de adquirir un producto. El cumplimiento de esta expectativa determinará el nivel de calidad percibida, sirviendo como referencia para calificarla como positiva o negativa.

Debido a la importancia y relevancia de la satisfacción del cliente se requiere de sistemas tecnológicos que permitan disponer de información organizada, Delgado (2015) indicó que los sistemas de apoyo proporcionan información necesaria para que los administradores tomen decisiones, así como satisfacer las necesidades de los directivos respecto a información general o de determinados departamentos.

La adopción de un sistema de información gerencial inicia partiendo de un diagnóstico de las necesidades de información de cada área específica, al igual que de sus proyectos y programas (Suárez, 2017). Este sistema se interrelaciona y comunica con todos los módulos para así lograr los propósitos definidos estando entre sus beneficios el acceso rápido a la información, evitar pérdidas de tiempo recogiendo información, solucionar el problema de falta de comunicación entre las diferentes instancias, etc.

Los sistemas de información son necesarios actualmente ya que analizan grandes cantidades de datos que contienen información relevante y sirven para tomar las decisiones necesarias, alcanzando con ello los objetivos de la institución (Isaza, 2018), la creación y aplicación de estos sistemas brinda apoyo para que la empresa pueda competir.

Entre las ventajas que Borja y Ferruzola (2015) indicaron respecto a estos sistemas están el hecho que permiten ajustar la maniobra gerencial para aumentar o disminuir el impacto de las decisiones y conseguir resultados. A su vez, agregan valor a la gestión después de dar soporte al manejo de los procesos; analiza la rentabilidad económica, social y financiera de la empresa; integra datos 


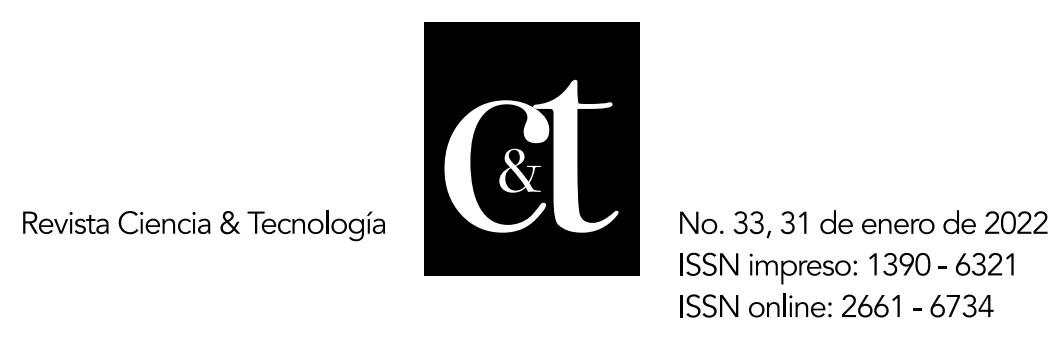

para dar información actualizada; y apoya a las funciones gerenciales de planeación, organización, dirección y control.

Por su parte, Pequeño (2015) expresó que las ventajas de estos sistemas implican el dar soporte más amplio a las tareas que se ejecutan dentro de una empresa y funcionan como un gestor de toma de decisiones. Como tal, impulsan la creación de grupos de trabajo ya que cada área debe recolectar información general sobre su función.

Entre los sistemas que guardan relación a las necesidades de los clientes, siendo en este caso el débil seguimiento durante y posterior a la venta que TEMCORPSA S.A realiza sobre sus clientes, se encuentran:

Microsoft Power BI, un servicio de análisis empresarial de Microsoft que proporciona visualizaciones interactivas en una interfaz simple que facilita su manejo para los usuarios finales (Microsoft, 2020).

Power Pivot, es el segundo sistema considerado funcionando como un complemento para Microsoft Excel también dirigido para el análisis de datos. Su uso crea modelos sofisticados de datos, combinándolos para presentar información rápida y compartirla con facilidad (Microsoft, 2020).

Tableu Partner, es el tercer sistema, utilizado en el análisis de datos, seguro y flexible que permite a quienes lo utilizan el ingreso de datos, además de permitir su análisis desde un dispositivo móvil o PC, presentando cálculos estadísticos, análisis avanzados y tablas que facilitarán la interpretación de datos, además de identificar patrones que permitan combinar la información de distintas áreas organizacionales (Xertica, 2020).

Dicho esto, el proyecto plantea como objetivo mejorar la calidad del servicio entregado a los clientes de la empresa TEMCORPSA S proponiendo un sistema de información gerencial para este caso.

\section{Metodología}

Sobre los tipos de investigación se incluyeron el documental y el de campo. En el estudio documental se revisó bibliografía que permitió comprender la situación planteada desde una perspectiva teórica. De esta forma se logró definir las variables de la investigación a la calidad del servicio al cliente y los Sistemas de información gerencial.

En lo que respecta a la investigación de campo involucró la interacción en la realidad objeto de análisis ayudó a identificar cómo el servicio proporcionado al cliente en la empresa TEMCORPSA S.A es percibido, las limitaciones existentes y cómo el Sistema de Información Gerencial puede contribuir a su calidad.

Así mismo, se emplearon los enfoques cuantitativos y el cualitativos. Con el primero, se permitió conocer la postura de individuos relacionados a la empresa TEMCORPSA, permitiendo evaluar la calidad del servicio al cliente, sus limitaciones, cómo un SIG podría influir en la calidad del servicio y otros puntos relacionados. Por otro lado, con el enfoque cualitativo se obtuvo información documental para describir las características del sistema Microsoft Power BI y sus funcionalidades, justificando su selección dentro de la investigación. 


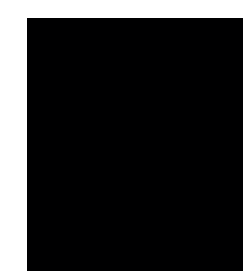

El tipo de muestreo aplicado fue no probabilístico, escogiéndose los individuos a consultar en función de su relevancia y facilidad de acceso para el estudio. Dicho esto, la muestra del talento humano en TEMCORPSA S.A fue el personal directivo, entre ellos el Gerente General, Gerente Financiero y Administrativo, Gerente de Logística y Operaciones, además del personal involucrado en el servicio al cliente, desde la atención inicial, el almacenamiento de la carga y su despacho.

Estos sumaron un total de 10 trabajadores considerados como individuos de interés para la investigación. En relación a los clientes, se pudo evidenciar que entre enero a octubre del año 2020 la empresa TEMCORPSA S.A mantuvo una cartera de conformada por 40 clientes. Bajo este muestreo no probabilístico a conveniencia se determinó que se consultarían al total de clientes registrado para evaluar la calidad percibida en torno al servicio.

De la investigación se obtuvo que la variable dependiente es la calidad del servicio al cliente y las variables Independientes fueron la calidad, la tecnología y el financiero.

La información se recolectó mediante encuestas, aplicándose a informantes clave. Para las encuestas aplicadas al talento humano de la empresa existió un contacto previo con cada individuo de interés para responder cada interrogante. En relación a los clientes, permitiendo los resultados medir la percepción de calidad respecto al servicio, las debilidades que existen en la empresa y cómo podría influir un SIG en la calidad.

En la encuesta para evaluar sus percepciones en función de la calidad del servicio recibido, identificando si cumple o no sus expectativas. Las respuestas se presentaron en escala de Likert entre 1 a 5 , en donde 1 hacía referencia a totalmente desacuerdo y 5 a totalmente de acuerdo

\section{Resultados y discusión}

\section{Análisis de la situación actual}

Con los resultados de la recolección de datos, se describe la realidad de la empresa TEMCORPSA S.A respecto a cómo ofrece el servicio al cliente, las debilidades existentes, la calidad percibida por el público en torno al servicio, entre otros aspectos. Los resultados se recopilaron a través de encuestas, tanto al talento humano de la empresa y a sus clientes.

\section{Resultados de la encuesta al talento humano}

En primer lugar, se presentan los datos recolectados mediante encuestas al talento humano, teniendo en cuenta que ascienden a 10 participantes.

Se presenta el análisis y discusión de los resultados obtenidos tras la interpretación de los datos arrojados por el instrumento. Los mismos, se recogen en tablas y/o figuras, mencionadas en el texto del trabajo. A continuación, algunos ejemplos: 


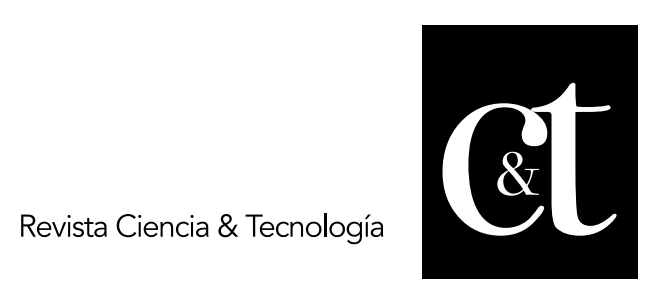

No. 33, 31 de enero de 2022

ISSN impreso: 1390 - 6321

ISSN online: 2661 - 6734

Fiabilidad del servicio.

1. En cada contratación, el cliente recibe fotografías de la carga cuando es almacenada

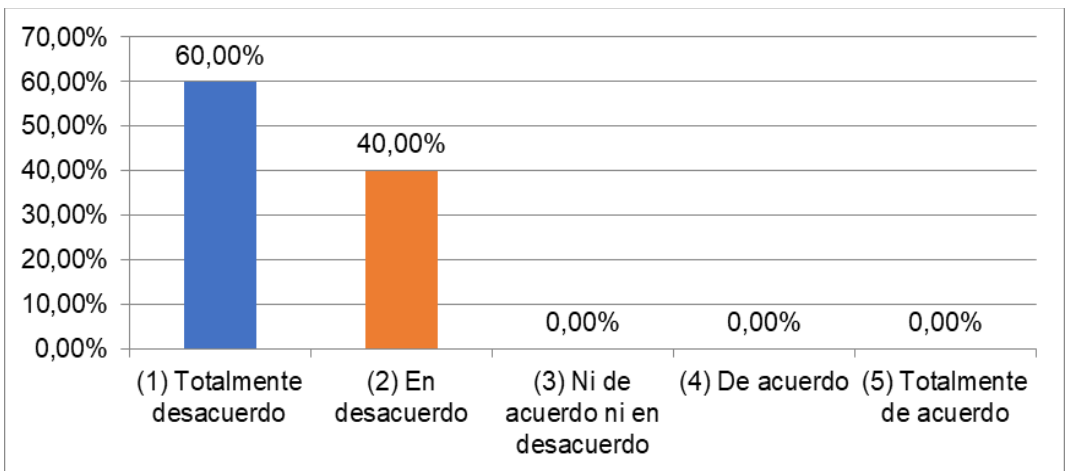

Figura 1: Recepción de fotografías de la carga en cada contrato Fuente: Cotto (2020)

Los resultados permiten evidenciar, según comentarios del talento humano, que los clientes no reciben un respaldo visual de su carga una vez es almacenada en las cámaras de frío. Estos les permitiría reducir la incertidumbre del cliente respecto a si la mercancía se colocó en óptimas condiciones y está segura, evitando que realice llamadas posteriores con este fin.

Seguridad del servicio.

2.En cada contratación, el cliente recibe un informe sobre el status de la carga

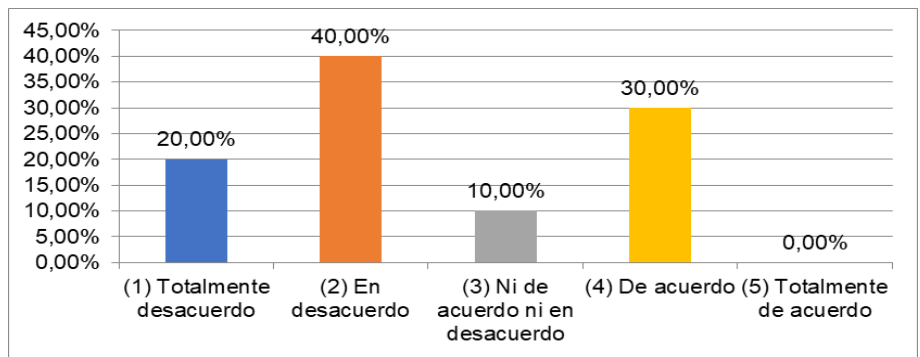

Figura 2. Recepción de informe sobre el estado de la carga Fuente: Cotto (2020)

Las respuestas del talento humano permiten denotar que es poco probable que un cliente reciba un informe sobre el estado de su carga una vez ha sido almacenada. Considerando que tampoco recibe fotografías de la mercancía, lo expuesto puede alimentar la inseguridad del cliente respecto a la integridad de los productos dentro de las cámaras de frío, aumentando aún más la probabilidad de que realicen llamadas para recibir información. 


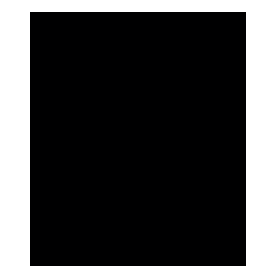

Capacidad de respuesta.

2. Existe un registro de clientes que permite pronosticar la demanda y anticiparse a sus necesidades

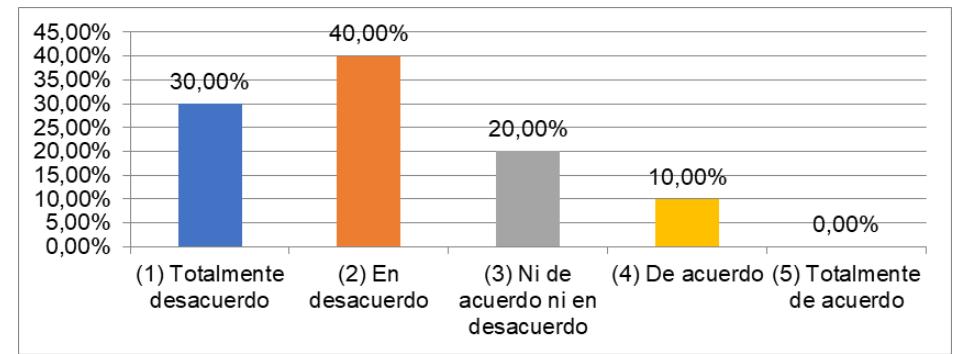

Figura 3. Registro de cliente

Fuente: Cotto (2020)

Para conocer en qué nivel la empresa puede anticiparse a los pedidos de potenciales clientes y programar mejor su servicio al público, se consultó si mantiene un registro de clientes. En respuesta a ello se pudo evidenciar que la empresa no dispone de esto, disminuyendo su capacidad de respuesta en brinda un servicio eficiente al público, contactando clientes y ofreciendo sus servicios para incrementar las ventas.

6. Existe intención para la adopción de algún sistema de información gerencial que permita analizar datos y obtener información necesaria para la mejora del servicio al cliente, de venta y post venta

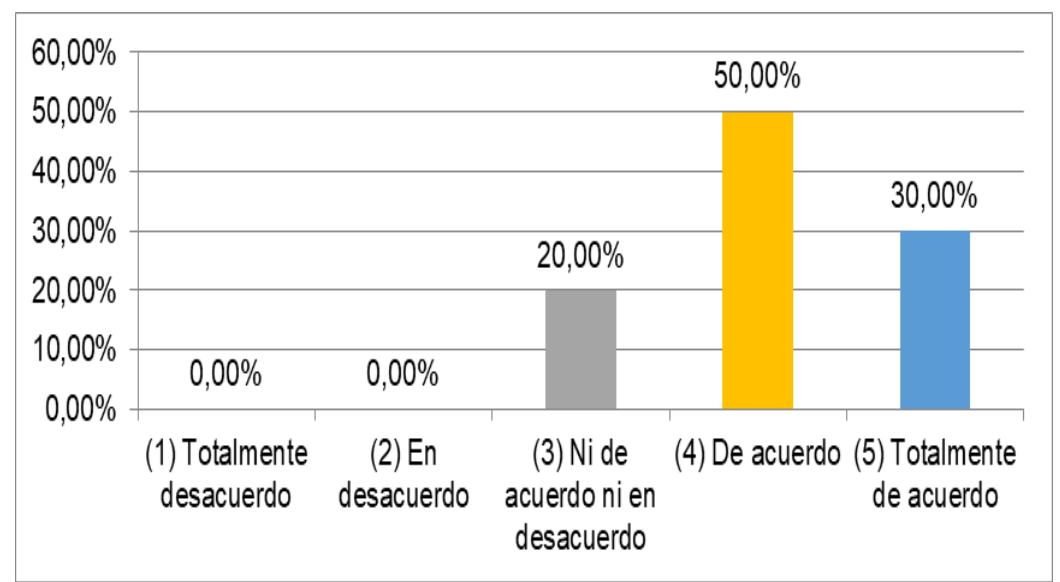

Figura 4. Intención en adoptar un SIG

Fuente: Cotto (2020)

Ante la debilidad de la empresa, siendo la carencia de una base de datos que fortaleza su toma de decisiones respecto a cómo brindar un servicio eficiente al público, el cual haga posible anticiparse a sus necesidades y favorecer a las ventas, 


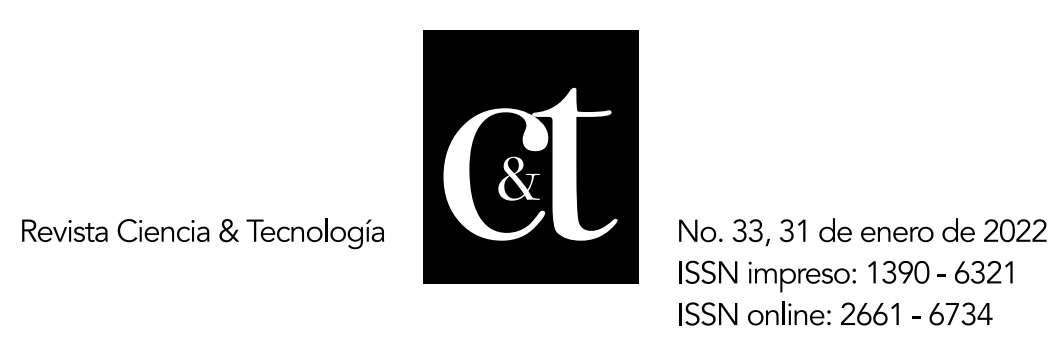

se consultó si existía intención de adoptar un SIG. La respuesta de encuestados permite suponer que existe tal intención, concentrándose las respuestas en "de acuerdo" y "totalmente de acuerdo".

\section{Resultados de la encuesta a clientes}

En esta sección se presentan los resultados de la encuesta a clientes, los cuales ascendieron a 40 individuos quienes habían accedido al servicio.

Fiabilidad del servicio.

1. Cumple lo prometido la empresa TEMCORPSA con respecto al servicio

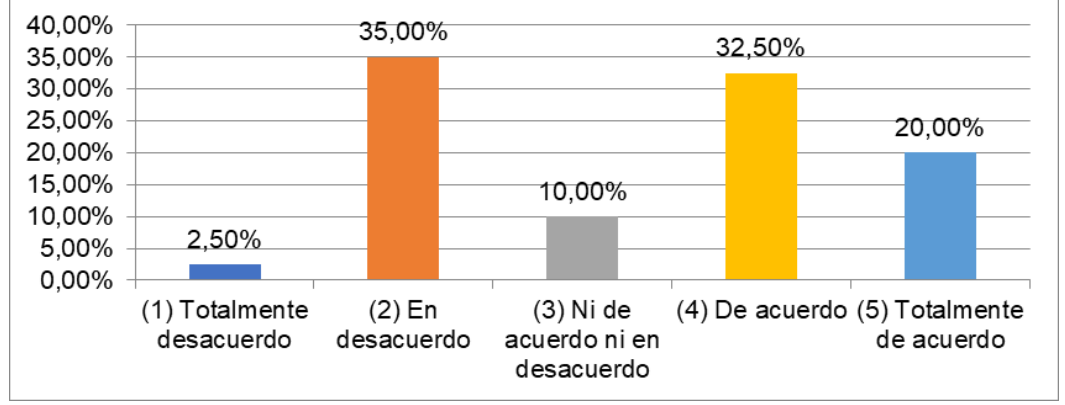

Figura 1. Culminación del servicio en el tiempo prometido Fuente: Cotto (2020)

Se evidencia que los clientes perciben, en su mayoría, que la empresa entrega la carga en el tiempo acordado, esto en un 52,50\% teniendo en cuenta que el $32,50 \%$ indicó estar de acuerdo y el $20 \%$ totalmente de acuerdo. Sin embargo, al analizar las respuestas individuales, el $35 \%$ se ubica en desacuerdo, permitiendo identificar que existe un grupo importante de clientes quienes indican que sus contratos no culminaron en el tiempo fijado, situación que puede motivar su insatisfacción en torno al servicio y percepción de mala calidad.

Seguridad del servicio.

2. No cometen errores al brindarle el servicio (su mercancía se almacena adecuadamente)

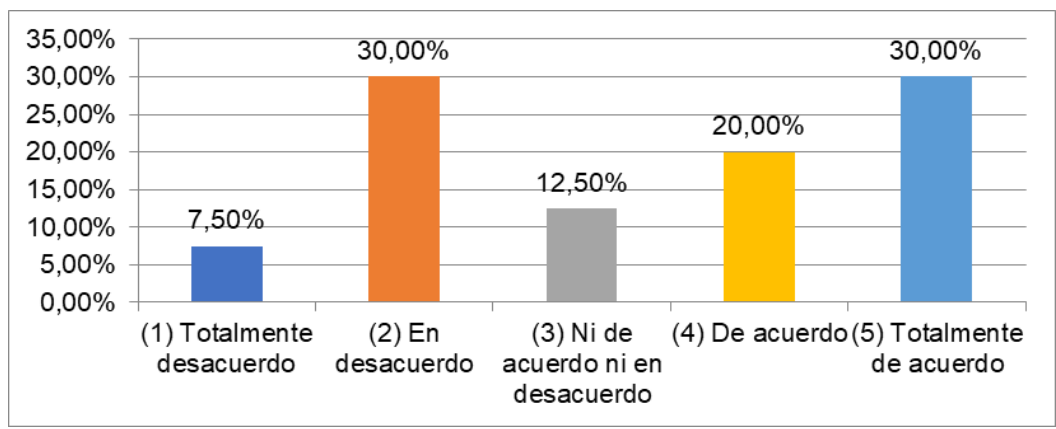

Figura 2. No cometen errores en el servicio

Fuente: Cotto (2020)

73

Véliz y Cotto. Sistema de información gerencial y su influencia en la calidad del servicio: empresa TEMCORPSA 


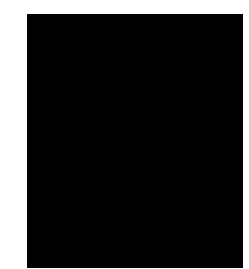

Existen opiniones divididas al respecto, considerando que un $30 \%$ indicó que estar totalmente de acuerdo que la empresa no comete errores, mientras otro $30 \%$ manifestó estar en desacuerdo. Aquello da a notar que existen casos cuando la empresa comete errores, lo cual ocasionará que el cliente percibe un servicio inadecuado que deteriorará su experiencia con la empresa.

3. Recibe algún respaldo (fotografías o informes) que brinde respaldo sobre la seguridad de su carga

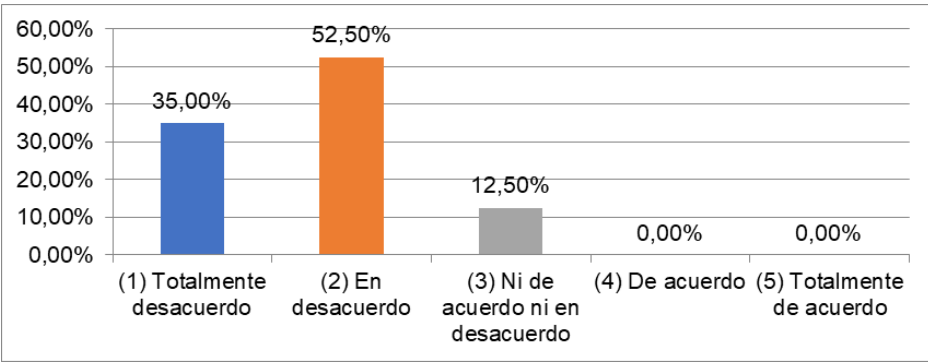

Figura 3. Recepción de algún respaldo de la carga almacenada

Fuente: Cotto (2020)

Sobre la recepción de algún respaldo de la carga, ya sean fotografías o informes sobre el estado de la misma, los clientes dieron respuestas negativas a esta interrogante. Es decir, que no las reciben, incrementando la incertidumbre en torno a si la carga se encuentra en condiciones adecuadas.

4. Siente, como cliente, que su mercancía está segura con TEMCORPSA

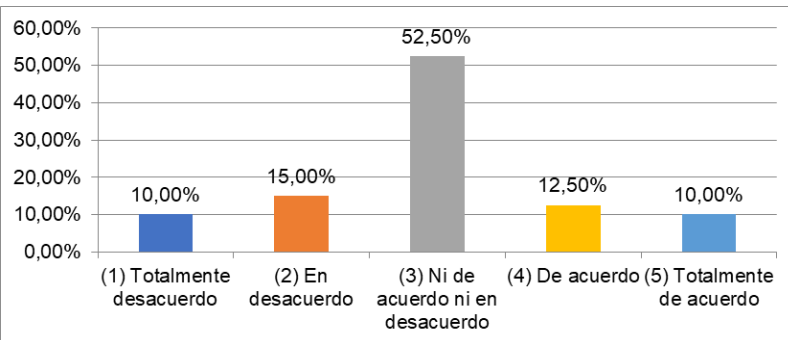

Figura 4. Seguridad de la mercancía Fuente: Cotto (2020)

Con las respuestas de los consultados es posible notar que consideran que la mercancía no está segura ni insegura en la empresa; sin embargo, las respuestas negativas presentan un mayor porcentaje frente a las positivas. Es decir, que también existen grupos de clientes quienes mencionan que la mercancía no está segura, es decir que la empresa no le transmite tranquilidad respecto a la carga almacenada. 


\section{저}

Empatía.

5. Se preocupan por los clientes y realizan un seguimiento continuo (venta y post venta)

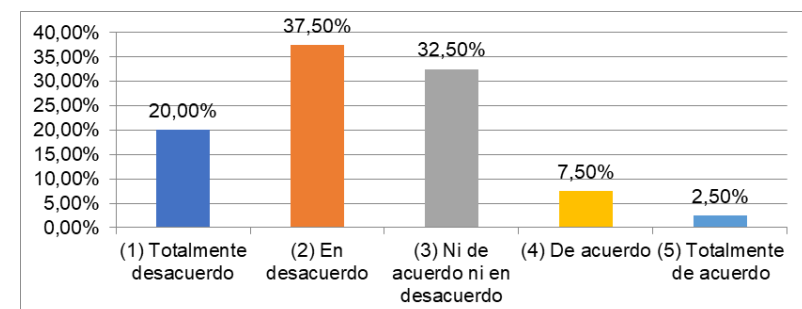

Figura 5. Preocupación por los clientes y seguimiento continuo

Fuente: Cotto (2020)

En base a las respuestas, se evidencia que los clientes tienen una percepción negativa respecto al seguimiento que la empresa realiza a su servicio, evidenciándose que la mayor cantidad de respuestas se ubican en desacuerdo con el $37,50 \%$. Para ello, resulta esencial que existan herramientas en la entidad que hagan posible darle un mejor seguimiento al cliente, logrando que el servicio cumpla sus expectativas al adaptar mejor la oferta.

\section{Análisis comparativo, evolución, tendencia y perspectivas}

La recolección de los datos de campo permitió conocer la perspectiva del talento humano y clientes de la empresa TEMCORPSA S.A. En primera instancia, puede mencionarse que la empresa no transmite la seguridad suficiente al cliente respecto al servicio que brindan, evidenciándose que no reciben fotografías o informes sobre el estado de la carga. Además, los trabajadores no transmiten mayor confianza, situación que puede deberse a errores previos con el servicio, mencionándose que han ocurrido problemas en la atención brindada al cliente.

Si bien, se realiza un mantenimiento óptimo de las cámaras de frío, el problema con el servicio se atribuye a otras causas, pudiendo notarse que los clientes perciben un escaso seguimiento durante la venta y post venta. Esto impide que la empresa pueda responder de forma efectiva a requerimientos del público, e incluso anticiparse al cliente y ofrecerle el servicio que necesita de forma personalizada. Contrario a ello, el servicio que se brinda al cliente no tiene esta característica de personalización, además la empresa no dispone de una base de datos o registro de clientes que le permita pronosticar la demanda, evaluar a los clientes según sus características, contactarlos y ofrecerle el servicio, incluso antes de que el cliente sea quien contacte con TEMCORPSA S.A, pudiendo también diseñarse promociones y otorgar beneficios mejor estructurados.

Lo mencionado ayudaría a planificar la oferta, optimizar el espacio en las cámaras de frío, además de llevar un mejor seguimiento de los clientes y, según la información almacenada, concentrarse en la búsqueda de nuevos clientes que puedan tener similares características y necesidades que los actuales. 
Contrario a ello, carece de herramientas tecnológicas que aporten a la mejora del servicio e incremento de las ventas, teniendo en cuenta que incluso las promociones y beneficios no cumplen los objetivos, no despertando suficiente interés en el público.

Se demuestra que existe un fuerte interés en la empresa por adoptar un SIG, el cual deberá ajustarse a las necesidades de la empresa, fortaleciendo el servicio mediante un mayor seguimiento a los contratos, reduciendo la desconfianza del cliente, aprovechando tecnologías para mantener así una comunicación más constante, evitando retrasos en los procesos y así cumplir las expectativas de cada cliente.

Además, con los datos, realizar análisis y obtener información sobre las necesidades de cada cliente, su comportamiento habitual y fortalecer la capacidad de respuesta de la empresa, aportando a las ventas y la mejora de la imagen organizacional. Con lo expuesto, considerando que previamente se evaluó el Software Microsoft Power BI en tres aspectos esenciales (calidad, tecnología y financiero), se indica que fue seleccionado para aportar a la solución de los problemas de TEMCORPSA S.A

\section{Conclusiones}

En relación del objetivo específico orientado a describir el proceso que involucra la atención y seguimiento al cliente dentro de la empresa, la investigación permitió determinar que en TEMCORPSA S.A se atienden a los clientes que demandan el servicio, sin una planificación previa que permita anticiparse a los requerimientos del público objetivo. Corresponde a identificar las limitaciones que mantiene el servicio al cliente externo proporcionado por TEMCORPSA S.A, comprobándose que están relacionadas a la seguridad que se transmite al cliente, el cumplimiento de las condiciones acordadas, la falta de un registro de clientes efectivo que aporte a la toma de decisiones orientadas a diseñar una oferta acorde a las expectativas del público, además de promociones y beneficios poco atractivos. Entre ellos se seleccionó el software Microsoft Power BI, el cual presenta un mejor desempeño en las variables tecnología, financiero y calidad frente a Power Pivot y Tableu Partner.

Para promover la mejora continua en la organización, esto específicamente en la calidad del servicio percibida por el cliente, debe aplicarse periódicamente el cuestionario desarrollado en este estudio para los usuarios externos. Dicha aplicación debe ser semestral a fin de evidenciar si existe o no mejoría en las percepciones del público, las debilidades que aún existen en el servicio y qué acciones deberían desarrollarse, de ser requeridas, para lograr su máxima satisfacción. Además, respecto al número de usuarios, se considera idóneo un total de cuatro que corresponderían al Gerente General, Gerente de Ventas, Jefe de Operaciones y Atención al cliente por estar más vinculados al servicio de alquiler de espacio en cámaras de frío. Que el servicio entregado al cliente externo involucre el envío de soportes visuales que permita a los contratantes constatar que la carga se encuentra almacenada correctamente, reduciendo la inseguridad percibida. 


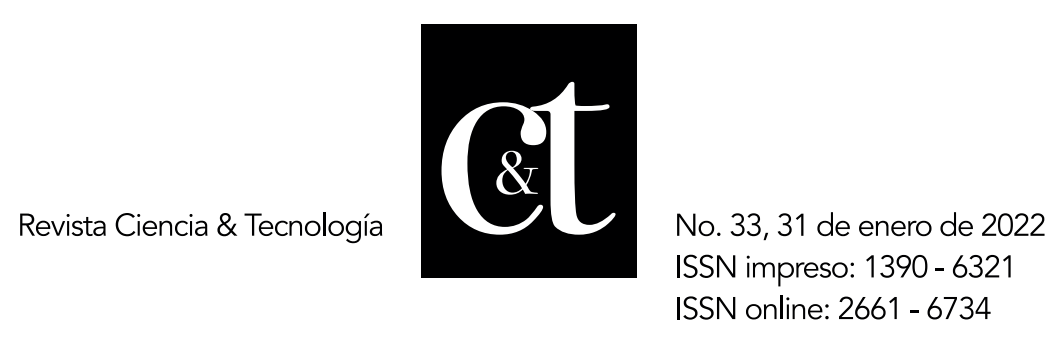

Además, es conveniente que se genere una base de datos de clientes, los cuales puedan ser contactos de manera anticipada para ofertarles el servicio. Diseñar incentivos y beneficios para los trabajadores de la empresa, además de desarrollar e implementar acciones que mejoren el ambiente laboral, lo cual motiven a los trabajadores a la entrega de un servicio de calidad al cliente.

\section{Referencias bibliográficas}

Alcaide, J. (2015). Fidelización de clientes. Madrid: ESIC.

Arenal, C. (2016). Calidad y servicios de proximidad en el pequeño comercio. UF2382. La Rioja: Tutor Formación.

Borja, E., \& Ferruzola, E. (24 de abril de 2015). Los sistemas de información gerencial: aplicabilidad en procesos empresariales y de educación superior en el Ecuador. Revista digitales UEPC, 1(8), 136-149.

Cortés, J. (2017). Sistemas de Gestión de Calidad (Iso 9001:2015). Málaga: ICB Editores.

Delgado, V. (2015). Planificando Estrategicamente! California: Lulu.com.

Hoces, L. (2018). Gestión de la captación de la clientela en la actividad de mediación de seguros y reaseguros. Madrid: IC Editorial.

Isaza, A. (2018). Garantía de la calidad en salud. Cómo organizar una empresa del sector salud. Barranquilla: Ediciones de la U.

Mateos, M. (2019). Atención al cliente y calidad en el servicio. COMM002PO. IC Editorial: Málaga.

Microsoft. (2020). Microsoft. Obtenido de Power Bi: https://powerbi.microsoft.com/es-es/

Pequeño, M. (2015). UF1884 - Almacenamiento de datos en sistemas ERP-CRM. Madrid: Editorial Elearning.

Sánchez, P. (2018). Fundamentos del plan de marketing en internet. COMM025PO. Málaga: IC Editorial.

Silva, J. (2018). La gestión y el desarrollo organizacional: Marco para mejorar el desempeño del capital humano. Alicante: 3 Ciencias.

Suárez, J. (2017). Sostenibilidad de las Organizaciones Sociales. Madrid: Chiado Editorial.

Vélez, C. (2018). Comercialización de productos y servicios en pequeños negocios o microempresas. Madrid: Elearning.

Xertica. (2020). Xertica. Obtenido de Tableu Partner: https://marketing.xertica.com/tableau xertica?utm term=software\%20business $\%$ 20intelligence\&utm campaign=TABLEAU+-+Adwords+-+Conversions++LATAM+-+Prospecting+-+Search+-+Non-

Brand/Broad\&utm source=adwords\&utm medium=ppc\&hsa net=adwords\&hsa grp $=105605115316 \&$ 